\title{
Comovimiento entre mercados accionarios de América Latina y Estados Unidos: Un enfoque de wavelets
}

\author{
Jesús Cuauhtémoc Téllez Gaytán* y Pablo López Sarabia**
}

\section{RESUMEN}

Este documento analiza la estructura de correlación entre los índices accionarios representativos de Estados Unidos como el S\&P500 y DJIA, así como de América Latina, como el IPC de México, IBovespa de Brasil y Merval de Argentina, para diferentes niveles de resolución y escalas de tiempo, que permite el enfoque de wavelets, contrario al enfoque tradicional basado en un análisis global de series de tiempo. Lo anterior se logra descomponiendo las series de rendimientos de los índices accionarios aplicando la transformada wavelet discreta de máximo traslape y como filtro la función de Daubechies de mínima asimetría MA (8). Los resultados empíricos muestran evidencia de un comportamiento no homogéneo entre las correlaciones de los mercados accionarios en horizontes de tiempo de diferente duración; en algunos casos la correlación es más fuerte en periodos con duración de muy corto plazo y en otros en periodos con duración de mayor plazo. La importancia de los resultados recae en la forma de estructuración de carteras con activos de diferentes mercados y diferentes horizontes de tiempo, tal que se obtengan diversificaciones más eficientes.

Palabras clave: transformada wavelet, descomposición por multirresolución, correlación, diversificación, mercados accionarios.

Clasificación JEL: C10, C63, G15.

\section{Abstract}

This document aims to analyze correlation structure among American equity indices such as S\&P500 and DJIA, in addition to the Latin American equity indices such as Mexico's IPC, Brazil's IBovespa and Argentina's Merval, at different resolution levels and time scales that is possible with wavelets approach, against the traditional approach related to a global analysis of time series. The time-scale decomposition of correlation is performed by decomposing equity indices returns using the maximal overlap discrete wavelet transform and the least asymmetric LA (8) Daubechies wavelet as filtering function. Results show a non-homogeneous correlation pattern among equity markets at different time scales: in some cases correlation showed a strong relationship at short-time scales duration and in others it showed a weaker one at long-time scales duration. The importance of this document concerns with asset allocation when making decisions in which market to invest and different time horizons, in such a way to perform efficient portfolio diversifications.

Key words: wavelet transform, multiresolution decomposition, correlation, diversification, equity market.

Classification JEL: C10, C63, G15.

\footnotetext{
* Profesor de la Facultad de Ciencias Económicas Administrativas, Universidad Autónoma del Carmen, Campeche: jctellezg@hotmail.com

** Profesor del Instituto Tecnológico de Monterrey, campus Estado de México: plopezs@itesm.mx
} 


\section{INTRODUCCIÓN}

Una de las principales herramientas estadísticas para la estructuración de portafolios de inversión, su administración y cuantificación del riesgo, es el análisis de varianza-covarianza la cual muestra, por medio de la varianza, el grado de variabilidad de la variable aleatoria, y de la covarianza el grado de comovimiento entre las variables aleatorias. Sus antecedentes en finanzas se registran en la teoría de portafolios de Markowitz (1952), la cual establece que el agente económico define como regla para estructurar sus carteras y seleccionar activos la relación media-varianza esperada de los rendimientos $(E-V)$, cuyos supuestos se basan en el modelo de caminata aleatoria.

A partir de la covarianza se obtiene el parámetro de correlación que mide el grado de asociación entre las variables aleatorias, el cual medirá la estructura de dependencia entre las variables siempre y cuando se cumpla el supuesto de normalidad. ${ }^{1}$ La aplicación común en finanzas del coeficiente de correlación se ha hecho en un contexto global, sin tener en cuenta los movimientos conjuntos que dicho estadístico puede indicar para diferentes niveles de frecuencias y escalas de tiempo de la serie de tiempo original. La importancia para las finanzas radica en que los rendimientos de los activos financieros se han caracterizado más por ser no estacionarios y de volatilidad cambiante en el tiempo, en lugar de independientes e idénticamente distribuidos.

El enfoque reciente aplicado a finanzas que ha considerado los fenómenos arriba citados se refiere al análisis por multirresolución utilizando wavelets (óndulas), análisis que permite la descomposición de la señal original en diferentes niveles de resolución y cada nivel de resolución asociado con una escala de tiempo. Las escalas de tiempo más bajas logran capturar los componentes de frecuencia más altos de la serie de tiempo que ocurren en periodos de tiempo muy cortos y las escalas de tiempo más altas permiten capturar los componentes de frecuencia más bajos que ocurren en periodos de tiempo muy largos. Lo anterior es posible transformando la serie original mediante funciones (base) especiales llamadas wavelets, que se representan a partir de aproximaciones sucesivas de series, similares a las series de Fourier, las cuales se representan por funciones seno y coseno.

\footnotetext{
${ }^{1}$ La teoría de cópulas especifica que el coeficiente de correlación (lineal) describe la dependencia entre variables aleatorias cuando las distribuciones marginales de las variables son gaussianas, y fuera de este contexto el coeficiente de correlación tiene poca validez para describir la estructura de dependencia (Nelsen, 2007).
} 
Las funciones wavelets tienen la propiedad de concentrar su energía en el tiempo para brindar un análisis de los fenómenos de temporalidad, no estacionariedad y volatilidad cambiante en el tiempo, tal como lo describen Burrus, Gopinath y Guo (1998). Las primeras aplicaciones en finanzas y economía las realizan Ramsey y Lampart (1999), quienes analizan las relaciones entre variables macroeconómicas tales como ingreso, consumo, tasa de interés y agregados monetarios.

En estudios posteriores, Lee (2004) analiza el comportamiento entre los mercados accionarios de Estados Unidos y Corea; Fernández (2005) estima la beta del CAPM (capital asset pricing model) y el valor en riesgo VaR-descompuesto para mercados accionarios emergentes. Norsworthy, et al. (2000) y Xiong, et al. (2005) igualmente estiman la beta del CAPM para emisoras del S\&P $500 \mathrm{y}$ de la bolsa de valores de Shangai, respectivamente. Lai, et al. (2006) estiman el riesgo de mercado de metales no férreos basado en un enfoque híbrido donde combinan wavelets y el modelo ARMA-GARCH; y Téllez, Vargas y Hernández (2009) estiman el $\mathrm{VaR}$ y la pérdida esperada en la cola del IPC utilizando principalmente la transformada wavelet discreta (TWD).

El presente documento tiene como objetivo estudiar el comovimiento en diferentes escalas de tiempo basado en la descomposición por multirresolución, entre principales mercados accionarios de América Latina y Estados Unidos, para lo cual se utiliza como filtro la función wavelet desarrollada por Daubechies (1988) de mínima asimetría y longitud ocho MA (8). ${ }^{2}$

Primero, se estiman los estadísticos descriptivos y correlaciones en una forma global, esto es, sobre la serie original de los rendimientos. Posteriormente, se descomponen las series de tiempo en diferentes niveles de resolución y se estiman las correlaciones entre los niveles de resolución para los diferentes pares de índices accionarios, por lo que existirán tantas matrices de correlaciones como niveles de resolución.

Por lo anterior, el documento se divide de la siguiente forma: la sección I describe primero el análisis por wavelets y enseguida revisa aplicaciones realizadas en finanzas y economía. La sección II describe los datos y metodología. La sección III presenta el análisis de los resultados. Finalmente se presentan las conclusiones.

\footnotetext{
${ }^{2}$ La aplicación de la función (discreta) de Daubechies MA (8) respecto de otras funciones wavelets se justifica en Härdle et al. (1998), al argumentar que ésta es una mejor aproximación a filtros de pase de banda ideales al permitir menor "fuga" de información.
} 


\section{REVISIÓN BIBLIOGRÁFICA}

\section{Análisis por wavelets}

Wavelets son funciones descritas por funciones base que conservan su característica oscilatoria y la capacidad para permitir simultáneamente el análisis de tiempo y frecuencia, por ello es posible procesar los datos en diferentes escalas y resoluciones e identificar en dónde una aparece frecuencia en particular en el dominio del tiempo. A. Graps (1995) comenta que el análisis por wavelets es como mirar el bosque (ventana grande) para detectar las grandes particularidades y a la vez mirar los árboles (ventanas pequeñas) para detectar las pequeñas particularidades.

Las funciones wavelets poseen dos propiedades que permiten su construcción conceptual y matemática: la admisibilidad y la regularidad. La propiedad de admisibilidad hace referencia al término de onda (wave), y consiste en que la transformada de Fourier de una función decaiga en la frecuencia cero; esto es, el valor promedio de una wavelet en el dominio del tiempo debe ser cero por lo que la función es oscilatoria y en consecuencia representa una onda. La condición de regularidad hace referencia al término let o decaimiento rápido, e implica que la función wavelet debe poseer suavización y concentración en el dominio del tiempo y frecuencia, por lo que la función wavelet tendrá $N$ momentos de desvanecimiento u órdenes de aproximación. Por tanto una wavelet es una función que oscila y decae con cierto número de desvanecimientos o aproximaciones.

La construcción de wavelets se basa en la dilatación $(W(x) \rightarrow W(2 x))$ y traslación $(W(2 x) \rightarrow W(2 x-1))$ de una función; si la dilatación y traslación se hace en forma infinita, se tendría la familia de funciones base $W_{j k}(x)=W\left(2^{j} x-k\right)$ en $L^{2}[0,1]$, que junto con una función de escala $\phi(x)$ representaría la construcción de una wavelet, donde $j \geq 0$ y $0 \leq k \leq 2^{j}$. Además, el producto interno de $\int \phi(x) W(x) d x$ es cero, teniendo como resultado la propiedad de ortogonalidad mutua entre las dilataciones y traslaciones de $W$. Lo anterior define la relevancia de las wavelets para analizar series de tiempo en diferentes escalas y frecuencias a lo largo del tiempo; es decir, la descomposición de la serie permite analizar a detalle sus características, y su reconstrucción (síntesis) para obtener la señal original. $^{3}$

\footnotetext{
${ }^{3}$ El concepto de escala, como lo ejemplifica Graps (1995), se refiere a las diferentes secciones en que se puede dividir una función base. En el supuesto inicial de una señal en el dominio de 0 a 1 , ésta se divide en funciones de dos etapas con rango de 0 a $1 / 2$ y de $1 / 2$ a 1 . Posteriormente, se vuelve
} 


\section{Wavelets en finanzas}

La importancia de estudiar el movimiento conjunto entre mercados accionarios en general y entre activos financieros en particular, se basa en la idea original de Markowtiz (1952) al considerar la importancia de las correlaciones entre activos en complemento con la cantidad de títulos que deben mantenerse en ellos. Una de las principales argumentaciones se refiere a que la reducción del riesgo es alimentada por la diversificación en activos cuyo movimiento no sea paralelo, a lo cual considera una diversificación eficiente. Grubel (1968) realiza una extensión de la idea original de Markowitz al argumentar que igualmente son posibles las ganancias en la riqueza mediante una diversificación internacional, con la que se buscaría que las correlaciones entre mercados fueran débiles para alcanzar una diversificación eficiente.

Derivadas de las ideas de Markowitz y Grubel, numerosas contribuciones se han realizado en el tema de diversificación, entre ellas, las de Levy y Sarnat (1970), Solnik (1974), Eun y Resnick (1991), French y Poterba (1991); otras consideran la cobertura por riesgo cambiario, como las de Adler y Simon (1986), Black (1989), Eun y Resnick (1988). Entre las contribuciones sobre diversificación en regiones considerando implicaciones de integración económica (NAFTA) se encuentran las de Griffin y Karolyi (1998), Kanas (1998), Flavin (2004), Brooks y Del Negro (2004), Aggrawal y Kyaw (2005), Berben y Jansen (2005), Darrat y Zhong (2005).

Al respecto, Forbes y Rigobon (1999) sostienen que el comovimiento entre mercados accionarios puede resultar en un contagio cuando el aumento en el comovimiento no es explicado por fundamentales como el vínculo comercial entre las economías. Adicionalmente, concluyen que a pesar de las épocas de crisis, como la del peso mexicano, se ha mantenido un vínculo fuerte entre los mercados, por lo que más allá de un contagio, lo que existe es interdependencia; por ello, una nueva línea de investigación es enfocarse en explicar por qué las economías son vulnerables a los movimientos en otros países. Un ejemplo del cuestionamiento de Forbes y Rigobon es la evidencia que muestra Ciner (2006) al estudiar el vínculo de los mercados accionarios del NAFTA, con el argumento de que el mayor comovimiento entre los mercados respectivos es el resultado de un auge global en las emisoras de tecnologías de información, más allá de una cambio permanente de la relación entre los mercados derivado del acuerdo comercial.

a dividir la señal original en funciones de cuatro etapas de 0 a $1 / 4,1 / 4$ a $1 / 2,1 / 2$ a $3 / 4$ y de $3 / 4$ a 1 . Cada conjunto de representaciones codifica la señal original en una escala o resolución particular. 
Sin embargo, Chukwuogor-Ndu y Kasibhatla (2007) cuestionan la mayor correlación que México presenta con Estados Unidos y establecen que este tipo de estudios (cointegración) son de largo plazo, por lo que los rendimientos accionarios en el corto plazo podrían diferir entre países y ser totalmente diferentes a una relación de largo plazo. En consecuencia, argumentan que dado el enfoque de estudio de largo plazo, sería ingenuo establecer que los beneficios de la diversificación internacional se eliminarán. Previamente a las conclusiones de Chukwuogor-Ndu y Kasibhatla (2007), Ramsey y Lampart (1997) argumentaron primero sobre un comportamiento no estacionario más allá de un proceso integrado de las series económicas, y en segundo término que las relaciones entre las variables económicas en diferentes escalas pueden diferir debido a los diferentes horizontes de tiempo.

Por ello, su estudio se basa en analizar las relaciones de diversas variables económicas utilizando el enfoque de wavelets. Uno de sus principales resultados, al aplicar pruebas de causalidad de Granger, es que en escalas de tiempo de duración corta, el ingreso causa al dinero y en escalas de mayor duración existe una causalidad bidireccional, por lo cual recomiendan prestar mayor atención a la modelación macroeconómica e incluir el desempeño de la escala de tiempo como una caracterización más de los datos económicos.

Posteriormente, Norsworthy, Li y Gorener (2000) aplican wavelets para estimar la beta del CAPM sobre emisoras pertenecientes al índice S\&P500; en su principal contribución al descomponer las series de los rendimientos de los activos en cinco niveles de resolución, encuentran que existe una mayor influencia del mercado sobre los rendimientos accionarios en los cambios de los componentes de frecuencias más altas (escalas de tiempo de menor duración), por lo que la eliminación del ruido en el mercado brindaría mejor información respecto de los movimientos de las emisoras en el largo plazo. En un estudio similar, Fernández (2005) descompone el CAPM en una versión internacional y estima el valor en riesgo $(\mathrm{VaR})$ para 7 países emergentes de América y Asia; su principal contribución reside en detectar que las pérdidas potenciales son mayores en horizontes de tiempo de menor duración que en un horizonte de largo plazo. En particular, encuentra que los mercados de mayor riesgo para invertir son Brasil, Indonesia y México, ya que la contribución marginal al riesgo en estos mercados fueron los mayores en escalas de tiempo de menor duración, relacionadas con los componentes de frecuencias más altas. En consecuencia, las posibilidades de diversificación considerando el factor escala de tiempo no serían las mismas para diferentes horizontes de inversión. 
Sin embargo, Atktan et al. (2009) encuentran que el CAPM para el mercado accionario de Estambul es un fenómeno multiescala cuyas predicciones son más significativas en el largo plazo, apoyando así los resultados de Norsworthy, Li y Gorener (2000). Téllez (2009) descompone las series de rendimientos de las emisoras pertenecientes al IPC y encuentra, así como Fernández (2005), que la mayor contribución marginal al VaR son aquellas frecuencias altas correspondientes a escalas de tiempo de menor duración. Sin embargo, al aplicar pruebas de backtesting descubre que la metodología de wavelets, en contraste con el modelo EWMA y de volatilidad condicionada GARCH, no resulta en el mejor ajuste para todas las emisoras en cuestión. Téllez, Vargas y Hernández (2009) estiman el $V a R$ y pérdida esperada para el IPC de México descomponiendo la serie en 7 niveles de resolución, y hallan que la metodología de wavelets al 95\% y $99 \%$ es apropiada para pronosticar las pérdidas esperadas.

En estudios previos de comovimiento, Lee (2004) estudia la relación de los mercados accionarios estadounidense y coreano, utilizando la función de Haar como principal filtro y encuentra que los efectos de transmisión de la volatilidad de los rendimientos accionarios en los seis niveles de resolución se da en dirección de Estados Unidos a Corea y nunca en sentido contrario. Sharkasi, Ruskin y Crane (2005) investigan con la misma metodología de Lee la relación entre los índices de Irlanda, Reino Unido, Portugal, Estados Unidos, Brasil, Japón y Hong Kong, y encuentran principalmente que el mercado estadounidense afecta a los asiáticos y éstos a Brasil y a los mercados europeos. Behrad (2008) construye tres portafolios con emisoras del NYSE, AMEX y NASDAQ, cuyas series de rendimientos descompone en tres niveles de resolución utilizando la función de Haar. Sus principales resultados son que las correlaciones de los activos y sus desviaciones estándar en cada portafolio difieren en cada escala y de aquella de la serie original. Por lo que, después de reducir el ruido de la serie original, la asignación de los pesos en los portafolios mejora y en consecuencia el rendimiento de los mismos.

Con base en la bibliografía revisada sobre la aplicación de wavelets, principalmente en finanzas, y partiendo de las ideas originales de Markowitz y Grubel, y en paralelo con las interrogantes que han surgido de las implicaciones que tiene la escala de tiempo en las relaciones de variables económicas y financieras, el presente documento tiene como finalidad estudiar la relación entre mercados accionarios de América Latina y Estados Unidos en diferentes escalas de tiempo, y contribuir así a la evidencia empírica sobre la diversificación internacional. 


\section{DATOS Y METOdología}

\section{Datos}

La información que utiliza este artículo son los índices semanales del Standard and Poor's (S\&P500), Dow Jones Industrial Average (DJIA), índice de precios y cotizaciones de México (IPC), índice de la bolsa de valores de Sao Paulo (IBovespa) y el índice del mercado de valores de Argentina (Merval) para el periodo del 9 de junio de 1991 al 27 de diciembre de 2009, por lo que se obtuvieron 997 observaciones de la agencia de información de Reuters.

Los índices se transformaron en rendimientos logarítmicos de la forma:

$$
\operatorname{Re} t_{t}=\ln \frac{P_{t}}{P_{t-1}} .
$$

Se utilizan datos semanales para evitar problemas de no sincronización por trabajar con precios de mercados en diferentes localidades. Los rendimientos de los precios de los índices son expresados en la moneda local de cada país con el propósito de considerar las posibilidades de cobertura ante la exposición al riesgo cambiario (Ciner, 2006, p. 342) y evitar problemas de convertibilidad de monedas derivado de las fluctuaciones en el tipo de cambio y los supuestos de la paridad poder de compra (Chukwuogor y Kasibhatla, 2007, p. 43). ${ }^{4}$

La descomposición por multirresolución se realizó utilizando el software Splus 8.0, mientras que las gráficas y estadísticos descriptivos fueron estimados en E-Views 7.0. Las pruebas de hipótesis para algunos estadísticos relevantes se realizaron a un nivel de significancia del 0.05 .

La figura 1 muestra la evolución de los rendimientos de los índices accionarios seleccionados en el periodo de estudio, y en ella se observan cambios importantes en el valor de los índices tanto en periodos de crisis como en periodos de recuperación de las bolsas.

En el cuadro 1 se muestran los estadísticos descriptivos de los rendimientos logarítmicos, donde se observa que en el periodo de estudio el IBovespa de Brasil fue el único mercado accionario analizado en ofrecer mayores rendimientos positivos que negativos vistos a través del valor del sesgo y con un desempeño promedio por arriba del resto de los mercados. Los cinco índices

\footnotetext{
${ }^{4}$ En estudios de cointegración, como el de Forbes y Rigobon (1999), se encuentra que los resultados no se ven alterados al emplear datos en una sola moneda y en moneda local de cada país.
} 
accionarios han reflejado un comportamiento leptocúrtico, aunque los índices DJIA y S\&P500 de Estados Unidos muestran evidencia de mayores valores extremos por el alto valor de la curtosis, situación explicada por la presencia de volatilidad cambiante y condicionada (Ruppert, 2004, p. 372). La prueba de Jarque-Bera (JB), rechaza la hipótesis nula de normalidad en los rendimientos semanales, dado un nivel de significación del 0.05 , resultado que se refuerza con el valor de curtosis que es mayor a tres.

Figura 1. Evolución de los rendimientos de los índices accionarios seleccionados
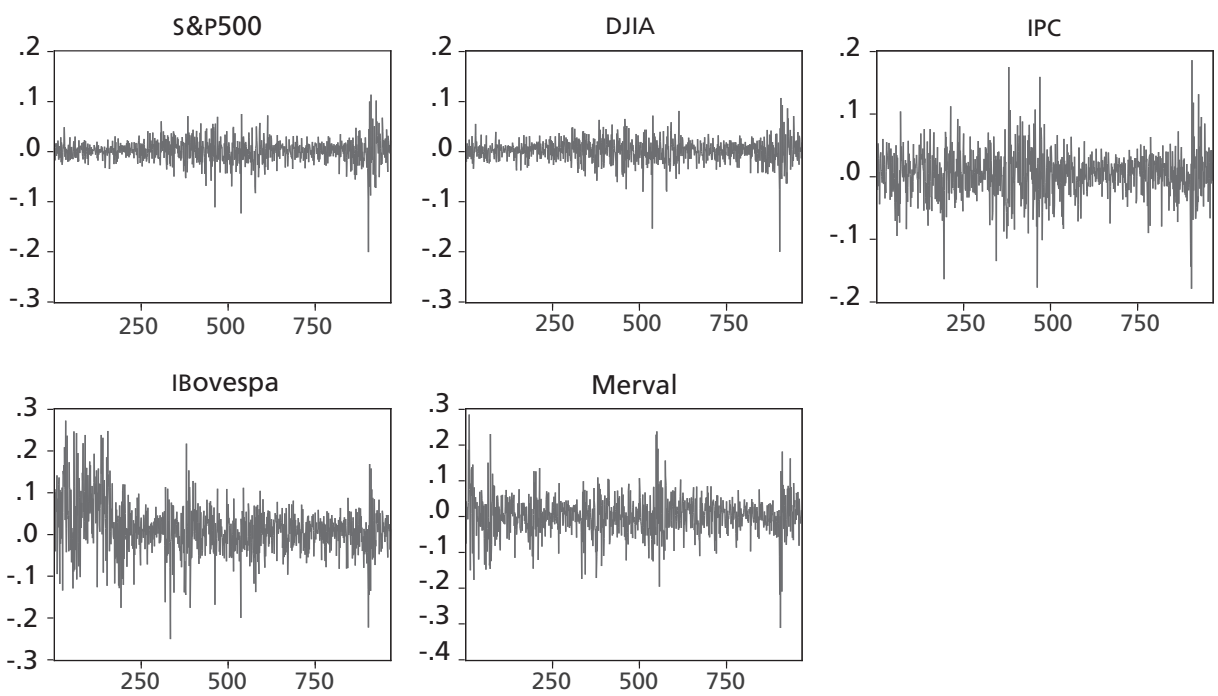

Fuente: Elaboración propia con datos de Reuters.

Cuadro 1. Estadísticos descriptivos de los rendimientos de los índices accionarios

\begin{tabular}{|l|c|c|c|c|c|c|c|c|c|}
\hline & Media & Mediana & Máximo & Mínimo & $\begin{array}{c}\text { Desv. } \\
\text { estándar }\end{array}$ & Sesgo & Curtosis & $\begin{array}{c}\text { Jarque- } \\
\text { Bera (JB) }\end{array}$ & $\begin{array}{c}\text { Probabi- } \\
\text { lidad (JB) }\end{array}$ \\
\hline S\&P500 & 0.001126 & 0.002358 & 0.113559 & -0.200837 & 0.023914 & -0.833651 & 10.82971 & 2579.391 & 0.0000 \\
\hline DJIA & 0.001307 & 0.002859 & 0.106977 & -0.200298 & 0.023445 & -0.942708 & 11.41354 & 2992.28 & 0.0000 \\
\hline IPC & 0.003489 & 0.005650 & 0.185786 & -0.179285 & 0.037498 & -0.207247 & 5.845589 & 332.8346 & 0.0000 \\
\hline IBovespa & 0.013748 & 0.011828 & 0.272682 & -0.250585 & 0.062221 & 0.366319 & 5.309685 & 236.3239 & 0.0000 \\
\hline Merval & 0.002141 & 0.004947 & 0.284993 & -0.311814 & 0.054893 & -0.089780 & 6.587767 & 519.3987 & 0.0000 \\
\hline
\end{tabular}

Fuente: Elaboración propia con datos de Reuters. 
El cuadro 2 muestra la matriz de correlaciones entre los cinco índices accionarios de manera global, pudiendo observarse que los índices IBovespa de Brasil y Merval de Argentina son los que menor grado de asociación han mantenido tanto con el mercado accionario estadounidense como con el mexicano. A pesar de que los resultados muestran correlaciones positivas, la debilidad de la correlación entre el mercado estadounidense y los de América Latina permite hacer una diversificación del riesgo, aunque ésta no sea la más eficiente.

Cuadro 2. Matriz de correlaciones entre los índices accionarios

\begin{tabular}{|l|c|c|c|c|c|}
\hline & S\&P500 & DJIA & IPC & IBovespa & Merval \\
\hline S\&P500 & 1 & 0.942260 & 0.593677 & 0.389501 & 0.399980 \\
\hline DJIA & 0.942260 & 1 & 0.562486 & 0.382294 & 0.398923 \\
\hline IPC & 0.593677 & 0.562486 & 1 & 0.429047 & 0.492902 \\
\hline IBovespa & 0.389501 & 0.382294 & 0.429047 & 1 & 0.402883 \\
\hline Merval & 0.399980 & 0.398923 & 0.492902 & 0.402883 & 1 \\
\hline
\end{tabular}

Fuente: Elaboración propia con datos de Reuters.

\section{Metodología}

Las series de rendimientos logarítmicas de manera global (señal original) se descomponen en niveles de resolución, también conocidos en la teoría de wavelets como cristales o detalles, utilizando la transformada wavelet discreta de máximo traslape (TWMT) y la función de Daubechies de mínima asimetría y longitud 8, donde la descomposición por multirresolución se representa de la forma:

$$
\begin{aligned}
& f(t)=\sum_{k} s_{J, k} \phi_{J, k}(t)+\sum_{k} d_{J, k} \phi_{J, k}(t)+\sum_{k} d_{J-1}, k \psi_{J-1}, k(t) \\
& +\ldots+\sum_{k} d_{1, k} \psi_{1, k}(t),
\end{aligned}
$$

donde $s_{J, k} \mathrm{y} d_{J, k}, \ldots, d_{l, k}$, son los coeficientes de la transformada wavelet contenidos en $\mathrm{W}$, los cuales miden la contribución de la función wavelet en la señal original. ${ }^{5}$ Los coeficientes $s_{J, k}$ son los coeficientes de suavización y representan

\footnotetext{
${ }^{5}$ La transformada wavelet es la transformada ortonormal en el nivel $J$ de un vector $\mathbf{X}, \mathbf{W}=\Omega \mathbf{X}$, donde $\mathbf{W}$ es un vector columna de longitud $N$, el cual contiene los coeficientes de la transformada: los primeros elementos $N-N / 2^{J}$ representan los coeficientes wavelet y los últimos elementos $N / 2^{J}$
} 
el comportamiento suave implícito en la señal a escalas gruesas (no refinadas) $2^{J} ; d_{j, k}$ son los coeficientes de detalle también conocidos como cristales, los cuales representan las desviaciones del comportamiento suave, donde $d_{J, k}$ describe las desviaciones en la escala gruesa y $d_{J 1, k}, \ldots, d_{1, k}$ son las desviaciones a escalas finas.

En general, la función $f(t)$ podrá representarse en términos de su serie de detalles en diferentes resoluciones:

$$
D_{j}(t)=\sum_{k} d_{j, k} \psi_{J, k}(t) \text { para } j=1,2, \ldots, J,
$$

y variaciones suaves:

$$
S_{J}(t)=\sum_{k} s_{J, k} \phi_{J, k}(t)
$$

teniendo como resultado:

$$
f(t)=S_{J}(t)+D_{J}(t)+D_{J-1}(t)+\ldots+D_{I}(t)
$$

lo cual es descrito igualmente como análisis por multirresolución. ${ }^{6}$

El estudio del comovimiento en diferentes horizontes de tiempo para efectos de este documento, hace referencia a la estructura de correlación entre los mercados accionarios seleccionados de América Latina y de Estados Unidos. Lo anterior se realiza estimando la correlación (wavelet) para cada par de índices accionarios a partir de las series originales descompuestas en niveles de resolución. La correlación wavelet se define como:

$$
\rho_{X}\left(\lambda_{j}\right)=\frac{\gamma_{X}\left(\lambda_{j}\right)}{v_{1}\left(\lambda_{j}\right) v_{2}\left(\lambda_{j}\right)}
$$

representan los coeficientes de escala; y, $\Omega$ es una matriz ortonormal $N$ x $N$ valuada en los reales, esto es, una matriz que satisface $\Omega^{T} \Omega=I$.

${ }^{6}$ El análisis por multirresolución vía transformada wavelet discreta de máximo traslape (TWDMT), es una versión modificada no diezmada de la transformada wavelet discreta (TWD), la cual funciona con cualquier tamaño de muestra $N$ sin limitarse a una longitud diádica de observaciones como lo es la TWD. Lo anterior hace que la TWDMT no sea sensible al punto inicial de la serie de tiempo, y ello se logra eliminando el método de submuestreo al aplicar doblemente el algoritmo piramidal de la TWD, tomando aquellos resultados descartados del filtro TWD mediante la filtración circularmente desplazada. Véase Gencay, Selcuk y Whitcher (2002). 
donde $v_{1}^{2}\left(\lambda_{j}\right), v_{2}^{2}\left(\lambda_{j}\right)$ son las varianzas wavelet de $x_{1, t}$ y $x_{2, t}$ asociadas a la escala $\lambda_{j}$. Asimismo, $\gamma_{X}\left(\lambda_{j}\right)$ corresponde a la covarianza wavelet que se define como:

$$
\gamma_{X}\left(\lambda_{j}\right)=\frac{1}{2 \lambda_{j}} \operatorname{Cov}\left(w_{1, j, t}, w_{2, j, t}\right),
$$

cuyo estimador insesgado, basado en la transformada wavelet discreta de máximo traslape (TWDMT) es:

$$
\hat{\gamma}_{X}\left(\lambda_{j}\right)=\frac{1}{\hat{N}_{j}} \sum_{l=L_{j}-1}^{N-1} \widetilde{w}_{1, j, l}, \widetilde{w}_{2, j, l},
$$

donde $\hat{N}_{j}=N-L_{j}+1$, y $\widetilde{w}_{1, j, l}$ y $\widetilde{w}_{2, j, l}$ son los coeficientes wavelet obtenidos por la TWDMT de $x_{1, t}$ y $x_{2, t}$, y contenidos en los vectores columna $W_{1}$ y $W_{2}$, respectivamente. $^{7}$

\section{ANÁLISIS DE LOS RESULTADOS}

La figura 2 muestra la descomposición por multirresolución (DMR) en los 7 niveles de resolución del IPC, cada detalle $D_{j}$ asociado a una diferente escala de tiempo y el componente de suavización $S_{j}$, donde el subíndice hace referencia al nivel de resolución (el anexo 1 muestra la descomposición por multirresolución de los demás índices accionarios seleccionados). El primer detalle o nivel de resolución $D_{1}$ captura los componentes de frecuencias más altas de los rendimientos del IPC que ocurren en el horizonte de tiempo más corto entre $\lambda_{1}=2^{j-1}=2^{0}=1$ y 2 semanas. El siguiente nivel $D_{2}$ muestra los componentes de frecuencia menores a los del nivel $D_{1}$ que ocurren en una escala de tiempo $\lambda_{2}=2^{2-1}=2^{1}=2$ a 4 semanas. En consecuencia, $D_{3}$ captura las frecuencias con duración de tiempo entre 4 y 8 semanas, $D_{4}$ las frecuencias entre 8 y 16 semanas, $D_{5}$ aquellas frecuencias que ocurren entre 16 y 32 semanas, y por último $D_{6}$ las que ocurren entre 32 y 64 semanas. $^{8}$

${ }^{7}$ Véase Gencay, Selcuk y Whitcher (2002) para un desarrollo más explícito de la correlación wavelet, y Serroukh, Walden y Percival (2000) para la varianza wavelet.

${ }^{8}$ Los valores que se observan en la figura 1 corresponden a valores reescalados, dado el proceso de transformación (convolución) que sufre la serie original con la función wavelet utilizando filtro. El eje vertical muestra los rendimientos reescalados según el nivel de resolución y el eje horizontal muestra el tiempo. Compárese el gráfico "IPC" de la figura 2 con aquel titulado igualmente como "IPC" de la figura 1, el cual presenta los rendimientos logarítmicos del IPC de México. 
Figura 2. Descomposición por multirresolución del IPC en 7 niveles
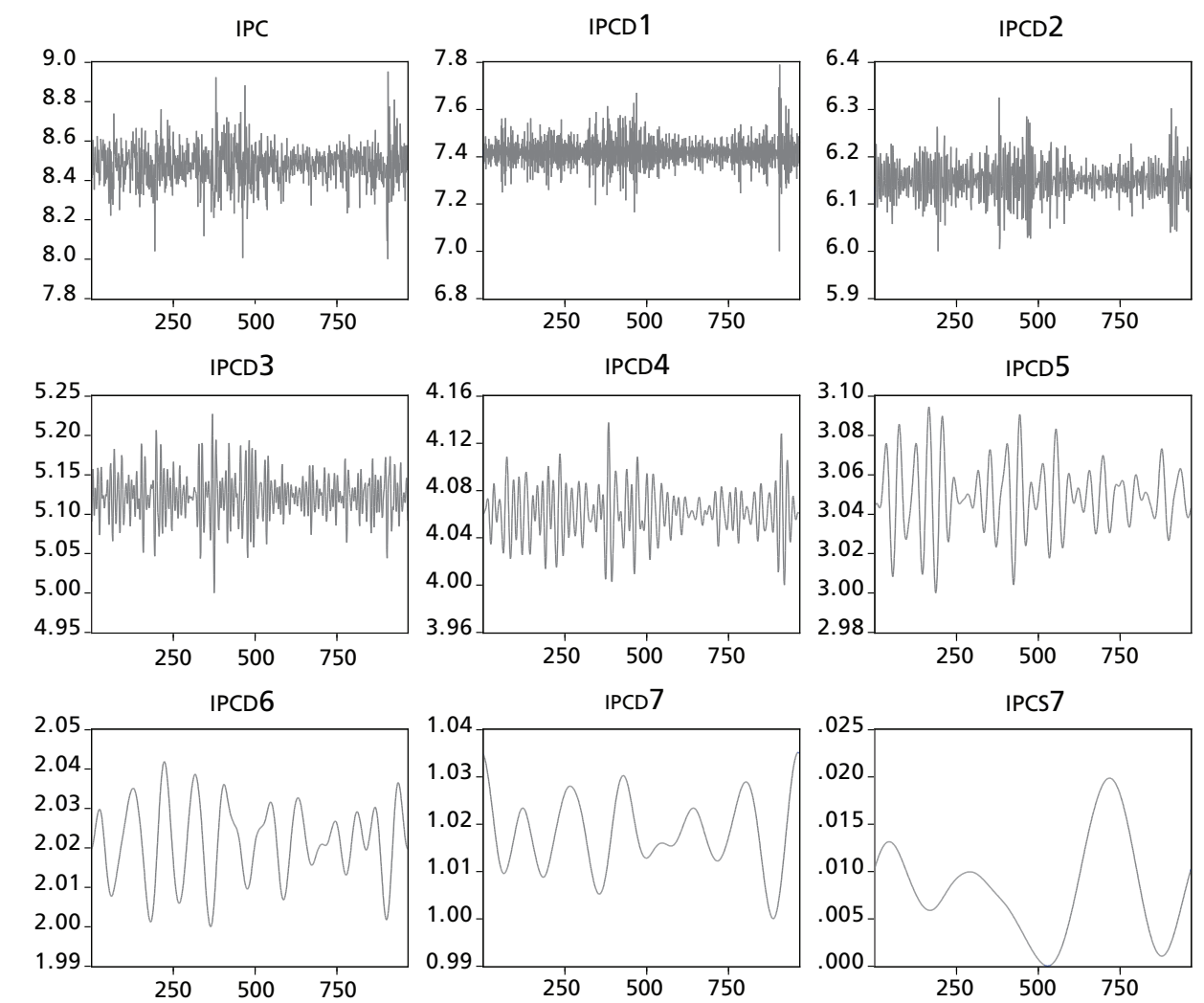

Fuente: Elaboración propia con datos de Reuters.

Una de las herramientas principales para determinar los niveles de resolución, o detalles apropiados para descomponer la serie original de los rendimientos, corresponde a la distribución de energía, la cual muestra la contribución que cada nivel de resolución representa de la señal original. El cuadro 3 muestra el porcentaje de energía que cada nivel de resolución captura de la serie de los rendimientos logarítmicos de los índices accionarios seleccionados, y permite apreciar que el nivel $D_{1}$ acumula aproximadamente $50 \%$ de la energía y es suficiente segmentar hasta el nivel de resolución $D_{7}$, ya que éstos acumulan una energía cercana a $99 \%$.

Con excepción del índice IBovespa de Brasil, en el cual se observa que los niveles de resolución intermedios, o escalas de tiempo de mediana duración, tienen poca contribución en la energía de la señal original, los niveles de resolu- 
ción equivalentes a escalas de tiempo superiores a 128 semanas concentradas en el componente $S_{7}$ tienen un peso significativo en la energía de la señal.

Cuadro 3. Distribución de energía de la DMR de los rendimientos de los índices accionarios seleccionados

\begin{tabular}{lcccccccc}
\hline Cristal & D1 & D2 & D3 & D4 & D5 & D6 & D7 & S7 \\
S\&P500 & 53.600 & 23.765 & 11.325 & 5.596 & 2.147 & 1.292 & 0.908 & 1.3607 \\
DJIA & 52.964 & 23.719 & 12.377 & 5.727 & 2.109 & 1.164 & 0.851 & 1.088 \\
IPC & 47.907 & 23.880 & 12.785 & 7.341 & 4.299 & 1.582 & 0.985 & 1.220 \\
IBovespa & 43.026 & 19.521 & 8.531 & 5.754 & 2.980 & 2.555 & 2.697 & 14.936 \\
Merval & 45.300 & 23.828 & 13.666 & 8.608 & 4.278 & 1.869 & 1.724 & 0.727 \\
\hline
\end{tabular}

Fuente: Elaboración propia con datos de Reuters.

La importancia de conocer la distribución de energía en cada escala de tiempo tiene sus implicaciones en finanzas, al estimar la varianza descompuesta en diferentes niveles de resolución. El cuadro 3 informa que los detalles con mayor energía corresponden al 1 y 2, por lo que los cambios más abruptos se están registrando en ventanas de 1 a 2 semanas y de 2 a 4 semanas. Lo anterior implica que la mayor variabilidad en los rendimientos de los índices accionarios en cuestión, proviene principalmente por los cambios registrados entre 1 y 2 , y 2 y 4 semanas. El cuadro 4 muestra la descomposición de la varianza (desviación estándar) para cada una de las series de los rendimientos accionarios, en donde se observa que la desviación estándar de los coeficientes wavelets disminuye significativamente a partir del segundo nivel de resolución, con excepción de Brasil, donde la desviación estándar es significativa en el horizonte mayor que 128 semanas derivado de la distribución de energía revisada en el cuadro 3.

Cuadro 4. Descomposición de la desviación estándar en 7 niveles de resolución Detalle

\begin{tabular}{lcccccccc}
\hline Índice & D1 & D2 & D3 & D4 & D5 & D6 & D7 & S7 \\
\hline S\&P500 & 0.050354 & 0.028880 & 0.019065 & 0.013483 & 0.008549 & 0.006558 & 0.005586 & 0.007354 \\
DJIA & 0.050143 & 0.028671 & 0.020201 & 0.013600 & 0.008463 & 0.006087 & 0.005526 & 0.006110 \\
IPC & 0.063497 & 0.038108 & 0.027515 & 0.020445 & 0.016948 & 0.009542 & 0.007626 & 0.005173 \\
IBovespa & 0.071049 & 0.040719 & 0.026342 & 0.022343 & 0.016128 & 0.013817 & 0.014269 & 0.036069 \\
Merval & 0.055198 & 0.034063 & 0.025136 & 0.020331 & 0.014657 & 0.008657 & 0.009277 & 0.006044 \\
\hline
\end{tabular}

Fuente: Elaboración propia con datos de Reuters. 
Los resultados generados por la descomposición mediante wavelets son relevantes para quienes tienen inversiones en exchange traded funds (ETF) y requieren información sobre determinados índices y su correlación para tomar sus posiciones, además de que los portafolios internacionales que intentan reproducir algunos índices pueden ser reequilibrados de mejor manera a lo largo del tiempo y con ello incrementar el valor del portafolio con la menor pérdida.

En particular, los resultados de una mayor correlación en dimensiones de corto plazo tienen un efecto directo en la determinación del valor en riesgo (VaR) de los portafolios que tienen activos ligados con los índices estimados o ETF, así que, a manera de ejemplo, la cuantificación del riesgo individual de activos financieros en el marco de valor en riesgo se reflejaría en mayores pérdidas potenciales para escalas de tiempo de menor duración respecto a escalas de tiempo de mayor duración; por lo que las frecuencias más altas y que ocurren en escalas menores serán las de mayor contribución marginal a la pérdida total esperada (Fernández, 2005, p. 16). ${ }^{9}$

\section{Análisis de la correlación wavelet}

El cuadro 5 muestra las correlaciones entre los índices accionarios en 7 niveles de resolución, cada uno de los cuales se relaciona con las diferentes escalas de tiempo. El patrón común que se observa es que las correlaciones entre mercados en diferentes escalas de tiempo no son homogéneas; en algunas escalas de tiempo cuya duración es corta el grado de asociación se fortalece, mientras que en detalles asociados a escalas con duración más larga la correlación se debilita. En el caso particular del Merval de Argentina, su correlación con el S\&P500 y el DJIA es fuerte en las primeras dos escalas de tiempo asociadas con duraciones de 1 a 2 y de 2 a 4 semanas; pero en una escala con duración de 4 a 6 semanas la correlación se debilita. Sin embargo, el grado de asociación se fortalece considerablemente cuando la escala es de 16 a 32 semanas y de 64 a 128 semanas. El valor que se observa de la correlación para duraciones mayores a 128 semanas se torna negativo.

Por ello, el comovimiento del Merval de Argentina con el mercado accionario estadounidense es totalmente heterogéneo, ya que es débil cuando las

\footnotetext{
${ }^{9}$ Un contraejemplo en que la mayor concentración de energía no siempre se presenta en los primeras escalas se refiere a Boroschek et al. (2002), quienes analizan las señales sísmicas en Chile y encuentran que la mayor distribución de energía se localiza en los detalles 4,5 y 6 , siendo el detalle 5 el de mayor concentración, con 38 por ciento.
} 
frecuencias son más altas y están asociadas con intervalos de tiempo de corta duración, mientras que la correlación se fortalece cuando las frecuencias son bajas y están asociadas con intervalos de tiempo intermedios, y pierde toda relación directa en el largo plazo.

Cuadro 5. Correlaciones entre índices accionarios por nivel de resolución

\begin{tabular}{|c|c|c|c|c|c|c|c|c|c|c|c|}
\hline D1 & SPX & DJI & IPC & IBOV & MERV & D2 & SPX & DJI & IPC & IBOV & MERV \\
\hline SPX & 1.0000 & 0.9443 & 0.6231 & 0.3845 & 0.4110 & SPX & 1.0000 & 0.9444 & 0.5721 & 0.4225 & 0.4470 \\
\hline DJI & 0.9443 & 1.0000 & 0.5985 & 0.3781 & 0.4204 & DJI & 0.9444 & 1.0000 & 0.5069 & 0.3941 & 0.4328 \\
\hline IPC & 0.6231 & 0.5985 & 1.0000 & 0.4344 & 0.4532 & IPC & 0.5721 & 0.5069 & 1.0000 & 0.4087 & 0.4580 \\
\hline IBOV & 0.3845 & 0.3781 & 0.4344 & 1.0000 & 0.3947 & IBOV & 0.4225 & 0.3941 & 0.4087 & 1.0000 & 0.3805 \\
\hline MERV & 0.4110 & 0.4204 & 0.4532 & 0.3947 & 1.0000 & MERV & 0.4470 & 0.4328 & 0.4580 & 0.3805 & 1.0000 \\
\hline D3 & SPX & DJI & IPC & IBOV & MERV & D4 & SPX & DJI & IPC & IBOV & MERV \\
\hline SPX & 1.0000 & 0.9453 & 0.5579 & 0.5110 & 0.2809 & SPX & 1.0000 & 0.9501 & 0.5667 & 0.5129 & 0.3717 \\
\hline DJI & 0.9453 & 1.0000 & 0.5281 & 0.5013 & 0.2679 & DJI & 0.9501 & 1.0000 & 0.5690 & 0.4979 & 0.3641 \\
\hline IPC & 0.5579 & 0.5281 & 1.0000 & 0.5530 & 0.5366 & IPC & 0.5667 & 0.5690 & 1.0000 & 0.4107 & 0.5646 \\
\hline IBOV & 0.5110 & 0.5013 & 0.5530 & 1.0000 & 0.4807 & IBOV & 0.5129 & 0.4979 & 0.4107 & 1.0000 & 0.5407 \\
\hline MERV & 0.2809 & 0.2679 & 0.5366 & 0.4807 & 1.0000 & MERV & 0.3717 & 0.3641 & 0.5646 & 0.5407 & 1.0000 \\
\hline D5 & SPX & DJI & IPC & IBOV & MERV & D6 & SPX & DJI & IPC & IBOV & MERV \\
\hline SPX & 1.0000 & 0.9289 & 0.6159 & 0.5919 & 0.6543 & SPX & 1.0000 & 0.9078 & 0.5472 & 0.0815 & 0.2978 \\
\hline DJI & 0.9289 & 1.0000 & 0.6609 & 0.6451 & 0.6620 & DJI & 0.9078 & 1.0000 & 0.6224 & 0.1984 & 0.4824 \\
\hline IPC & 0.6159 & 0.6609 & 1.0000 & 0.7183 & 0.6810 & IPC & 0.5472 & 0.6224 & 1.0000 & 0.7016 & 0.6777 \\
\hline IBOV & 0.5919 & 0.6451 & 0.7183 & 1.0000 & 0.5710 & IBOV & 0.0815 & 0.1984 & 0.7016 & 1.0000 & 0.6791 \\
\hline MERV & 0.6543 & 0.6620 & 0.6810 & 0.5710 & 1.0000 & MERV & 0.2978 & 0.4824 & 0.6777 & 0.6791 & 1.0000 \\
\hline D7 & SPX & DJI & IPC & IBOV & MERV & S7 & SPX & DJI & IPC & IBOV & MERV \\
\hline SPX & 1.0000 & 0.9822 & 0.7566 & 0.2212 & 0.6918 & SPX & 1.0000 & 0.9780 & 0.6118 & 0.1930 & -0.0030 \\
\hline DJI & 0.9822 & 1.0000 & 0.7892 & 0.2637 & 0.6889 & DJI & 0.9780 & 1.0000 & 0.5171 & 0.2389 & -0.0808 \\
\hline IPC & 0.7566 & 0.7892 & 1.0000 & 0.5706 & 0.8930 & IPC & 0.6118 & 0.5171 & 1.0000 & 0.2431 & 0.5434 \\
\hline IBOV & 0.2212 & 0.2637 & 0.5706 & 1.0000 & 0.5131 & IBOV & 0.1930 & 0.2389 & 0.2431 & 1.0000 & 0.0740 \\
\hline MERV & 0.6918 & 0.6889 & 0.8930 & 0.5131 & 1.0000 & MERV & -0.0030 & -0.0808 & 0.5434 & 0.0740 & 1.0000 \\
\hline
\end{tabular}

Fuente: Elaboración propia con datos de Reuters.

El fenómeno no homogéneo es distinto entre el Merval de Argentina y el IPC de México, pues en la medida que la escala de tiempo es mayor, ambos mercados muestran un comovimiento más fuerte, registrando hasta un grado de asociación de casi 0.90 en el horizonte de 64 a 128 semanas. Sin embargo, en 
periodos mayores a 128 semanas el grado de asociación se debilita. En el caso de la correlación del IPC de México con el mercado estadounidense, la asociación es relativamente fuerte para fluctuaciones más altas con duraciones muy cortas, pero cuando las fluctuaciones son muy bajas con duraciones largas, el grado de asociación se fortalece entre ambos mercados.

Los resultados previos muestran que no prevalece la misma estructura de correlación entre los mercados accionarios seleccionados para diferentes frecuencias y escalas de tiempo, tomando como marco de referencia el grado de asociación estimado entre las series de rendimientos logarítmicos en su forma global. En un estudio similar, Ranta (2008) encuentra que las correlaciones entre las volatilidades de los principales índices accionarios mundiales son débiles en escalas de tiempo menores, por lo que la diversificación resultaría más eficiente en horizontes de inversión muy cortos de tiempo.

Los resultados empíricos en este artículo muestran que la diversificación puede ser eficiente en ciertas duraciones, mientras que en otras la posibilidad de pérdidas fuertes puede ser mayor. Resulta, por ejemplo, que la diversificación entre el mercado mexicano y el brasileño es más eficiente en periodos de entre 1 y 2 semanas que en horizontes más largos, como aquellos de entre 16 y 32 semanas. Sin embargo, una diversificación más eficiente entre estos dos mercados resultaría mejor en periodos muy largos, cuyas duraciones fueran mayores a las 128 semanas.

\section{CONCLUSiOnes}

La flexibilidad de las wavelets para permitir el análisis de una serie de tiempo en el dominio de la frecuencia y el tiempo -contrario al análisis de Fourier, el cual únicamente indica la existencia de las frecuencias sin importar cuándo los componentes de las frecuencias aparecen-, ha motivado su aplicación en economía y finanzas para analizar y reexaminar ideas originales en cuanto al comportamiento entre las variables. En este sentido, Ramsey y Lampart (1997) argumentan sobre la factibilidad de que las relaciones de variables económicas cambien en diferentes escalas de tiempo ante la situación de que los agentes económicos se comportan de diferente forma según los intervalos de tiempo. Al respecto, reexaminan ideas originales de consumo-ingreso, dinero-ingreso y de bienes duraderos y no duraderos con la tasa de interés.

La aplicación de wavelets a las finanzas, considerando como punto de referencia las argumentaciones de Ramsey y Lampart (1997) y la particularidad 
de las series financieras por caracterizarse como no estacionarias, ha generado estudios principalmente en la estimación del riesgo sistemático y el comportamiento entre mercados financieros. Tal como se revisó en este documento, Norsworthy, Li y Gorener (2000) son considerados los pioneros en estos estudios, al estimar el CAPM para emisoras pertenecientes al Dow Jones, ante lo cual concluyen que la filtración o eliminación del ruido permite un mejor análisis del comportamiento de largo plazo de las emisoras. En cuanto al comportamiento de mercados financieros, Lee (2004) realiza su análisis de comovimiento entre mercados accionarios descomponiendo las series de rendimientos y aplicando regresiones sobre las mismas para identificar el mecanismo de transmisión de la volatilidad del mercado accionario estadunidense y coreano, cuyos resultados sostienen que la dirección de la información es de Estados Unidos a Corea para los diferentes horizontes de tiempo.

Este documento aplicó el análisis por wavelets para estudiar la estructura de correlación en diferentes horizontes de tiempo entre principales índices accionarios de América Latina y Estados Unidos, a partir de la descomposición por multirresolución usando la transformada wavelet discreta de máximo traslape de los rendimientos accionarios en diferentes niveles de resolución o detalles, y como principal filtro se utilizó la función de Daubechies de mínima asimetría y longitud 8. En general, los resultados arrojaron evidencia de correlación no homogénea entre los mercados accionarios, entendiendo en este documento el concepto de no homogeneidad como un patrón desigual de la correlación en diferentes escalas de tiempo. Se observó que los mercados estudiados no guardan la misma estructura de correlación en su forma global que en la forma detallada o descompuesta en niveles de frecuencia-escala.

En particular, se observó que la varianza estimada para cada mercado accionario es mayor en ventanas con menor duración que en aquellas de mayor duración. Las implicaciones de estos resultados en el marco de valor en riesgo de activos individuales corresponderían a observar mayores pérdidas potenciales en horizontes de muy corto tiempo respecto de aquellos de mayor plazo. Por ello, una política de administración de riesgos consideraría estrategias de reservas de capital según las pérdidas en diferentes horizontes de tiempo. Dicho argumento se sustenta con los resultados obtenidos por Fernández (2005) y al comentar que las frecuencias más altas que ocurren en periodos de muy corto tiempo son las de mayor contribución marginal a las pérdidas potenciales.

Respecto a los resultados de la estructura de correlación, la evidencia mostró que existen parejas de mercados cuyo grado de asociación es débil en 
periodos de corta duración y se fortalece en periodos de larga duración. Tal fue el caso de México con Estado Unidos, en el que la correlación IPC-DJI aumenta de 0.5985 a 0.7892 , aunque en cierta escala de tiempo en particular disminuyó a 0.5069. En otros casos, la correlación entre los mercados mostró ser más fuerte en periodos intermedios, como entre Brasil y Estados Unidos, y hasta negativa en periodos de largo plazo, como Argentina y Estados Unidos.

Los anteriores resultados tendrían sus implicaciones en la estructuración y administración de carteras de la siguiente forma: primero, en materia de teoría de portafolios, conforme a Markowitz (1952), los agentes económicos buscan la diversificación de carteras a partir de la reducción del riesgo, seleccionando activos cuyos movimientos no tengan la misma dirección o al menos que el grado de comovimiento no fuese alto. Derivado de ello, Markowitz señala que la importancia en la diversificación reside tanto en asignar recursos en los activos como en la correlación que hay entre ellos, por lo que la diversificación sería eficiente en tanto las correlaciones (históricas) fuesen bajas, denominando a esta técnica "análisis de media-varianza". Por otro lado, Grubel (1968) extiende el concepto de diversificación de Markowitz a un contexto internacional, argumentado que la diversificación internacional de portafolios es la fuente de mayores ganancias en la riqueza. Posteriormente, Eun y Resnick (1988) argumentan que la cobertura del riesgo cambiario incrementaría las ganancias de la diversificación internacional. En esta perspectiva de la diversificación internacional, algunos estudios se han centrado en analizar las posibilidades de diversificación en regiones cuyos países muestran integración económica y financiera, tal como lo hacen Fadhlaoui et al. (2009) al encontrar que la correlación es baja entre países emergentes y desarrollados del centro de Europa.

Por lo anterior, los resultados del presente estudio mostrarían, al utilizar las series de índices accionarios en su forma global, que en América existe posibilidad de diversificación internacional eficiente al observar que las correlaciones históricas son bajas. Sin embargo, una diversificación más eficiente podría obtenerse si se considera el ritmo de inversión, en el cual el tiempo de permanencia en los diferentes mercados contribuiría a mayores ganancias. Por ejemplo, en el caso de Argentina y Estados Unidos, en el que una diversificación eficiente se lograría con ventanas de tiempo de 4 a 8 semanas y de 32 a 64 semanas; mejor aún, en el largo plazo en horizontes de inversión mayores a 128 semanas se obtendría una mejor ganancia en la diversificación ya que en este horizonte el grado de comovimiento o correlación mostró ser negativa. Sin embargo, deberá prestarse atención en aquellos detalles donde la correlación es alta 
y de inmediato disminuye significativamente, como el caso de Brasil, en el cual con una ventana de 32 a 64 semanas la correlación mostró ser relativamente fuerte pero en la ventana siguiente, de 64 a 128 días, el grado de comovimiento disminuyó a casi cero, siendo este horizonte apropiado para mejorar la eficiencia de la diversificación.

Por tanto, y así como en el enfoque de inversiones top-down, al considerar el tiempo de permanencia en los diferentes mercados accionarios se realizaría una primera "gran toma" y después se fraccionaría en detalles más finos; la "gran toma" corresponde al enfoque (global) tradicional de analizar el comovimiento entre las variables económico-financieras y el enfoque wavelets permite fraccionar la "gran toma" para captar los detalles finos. Dicho análisis complementaría la visión clásica de la administración de portafolios de inversión considerando el factor "ritmo" relevante en la diversificación.

Complementando el análisis de los resultados de la varianza arriba descritos, en materia de administración de riesgos debe tenerse precaución al cuantificar el riesgo de un portafolio internacional, ya que los mercados accionarios al no tener el mismo grado de movimiento conjunto en diferentes horizontes de tiempo obtendrían menores posibilidades de reducir el riesgo y mayores pérdidas potenciales. Finalmente, los mismos resultados tendrían sus implicaciones en la valuación de activos, ya que dos activos que presumiblemente tuviesen una correlación alta, al omitirse los detalles más finos que brindasen información específica de las correlaciones en diferentes horizontes de tiempo, podrían tener sus efectos en precios de referencia distorsionados y posibilidades de arbitraje extraordinarias.

Este documento se limitó en analizar el comovimiento a partir de la estructura de correlación entre diferentes mercados accionarios en diferentes escalas de tiempo, utilizando la descomposición por multirresolución mediante la transformada wavelet discreta de máximo traslape. El estudio puede extenderse para analizar la coherencia de la correlación a lo largo del tiempo y detectar principalmente el grado de asociación y su significación en periodos de crisis y estables, utilizando la coherencia wavelet, como lo hacen Rua y Nunes (2009), quienes analizan el comovimiento entre los mercados accionarios de Alemania, Japón, Reino Unido y Estados Unidos, así como la estimación del valor en riesgo. Ranta (2010), por su parte, examina el fenómeno del contagio entre yen japonés, libra esterlina y marco alemán respecto al dólar estadounidense, en tanto Grinsted, Moore y Jevrejeva (2004) estudian series de tiempo geofísicas. Asimismo, el estudio puede realizarse considerando rendimientos accionarios ex- 
presados en una misma moneda y con periodicidad distinta a la semanal para evaluar la resultados obtenidos en el documento.

La importancia del estudio reside en contribuir a la evidencia empírica más que teórica de las implicaciones que guarda analizar los mercados accionarios bajo una "lupa grande" en contraste con fraccionar la toma grande en detalles finos. Estudios previos se habían realizado para examinar mercados desarrollados, como Estados Unidos, Europa y algunos emergentes de Asia y Medio Oriente, y este es el primero en analizar el comovimiento para mercados accionarios de América Latina y Estados Unidos.

\section{ANEXO 1}

Figura A1. Descomposición por multirresolución del S\&P500 en 7 niveles
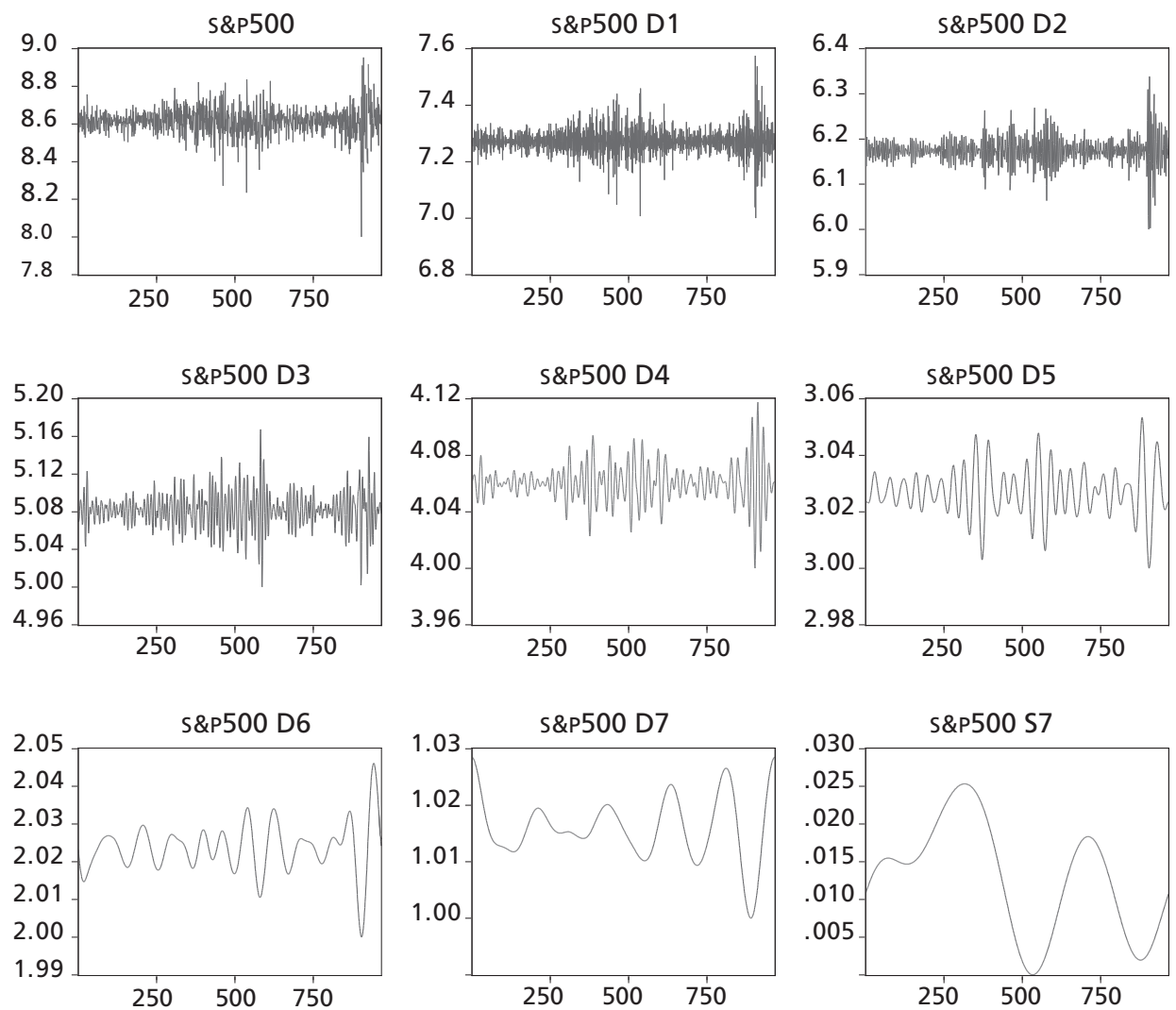
76 ECONOMÍA: TEORÍA Y PRÁCTICA • Nueva Época, número 32, enero-junio 2010

Figura A2. Descomposición por multirresolución del DJIA en 7 niveles
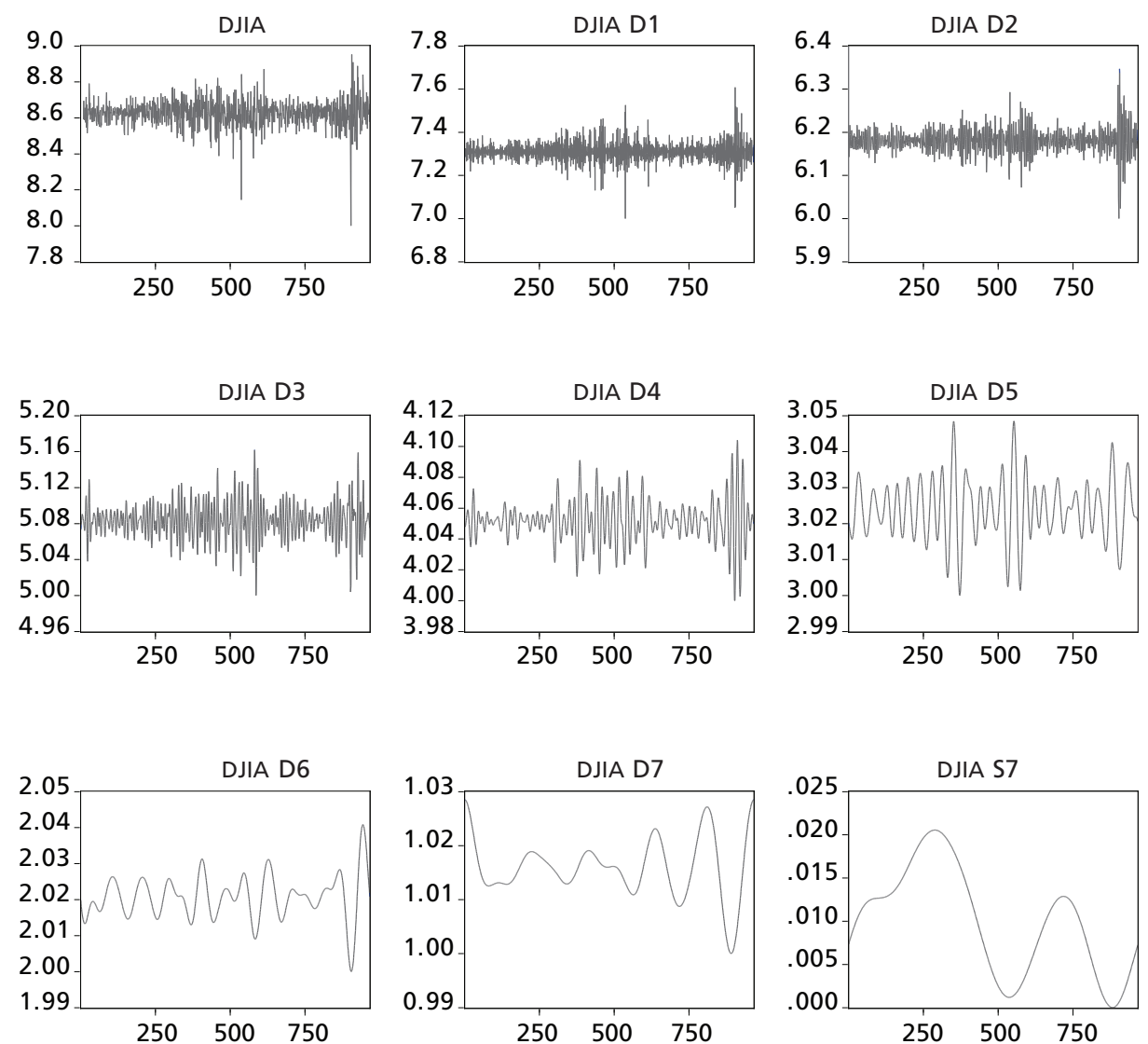
Figura A3. Descomposición por multirresolución del ıвovespa en 7 niveles
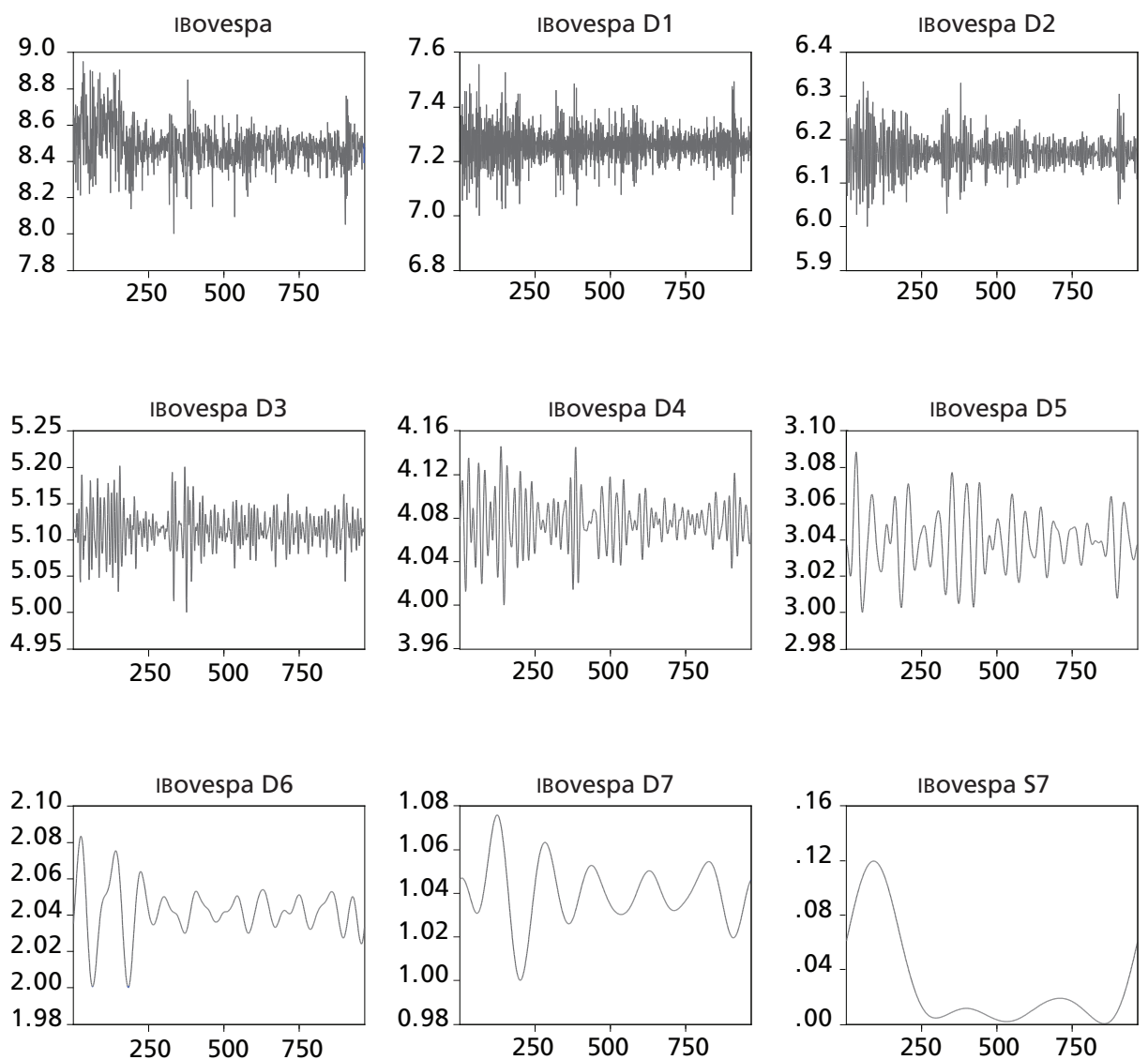
78 ECONOMÍA: TEORÍA Y PRÁCTICA • Nueva Época, número 32, enero-junio 2010

Figura A4. Descomposición por multirresolución del Merval en 7 niveles
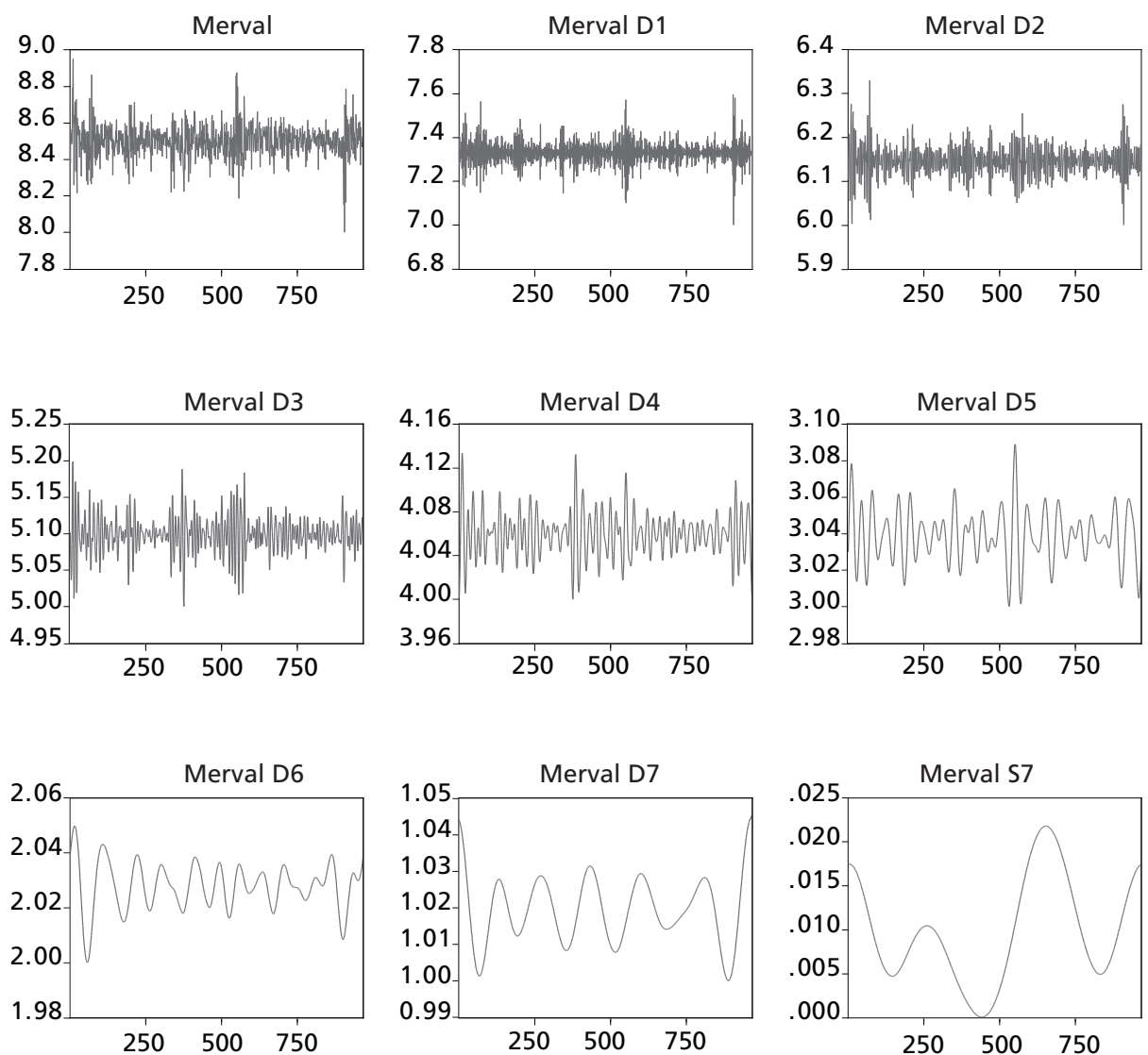

Fuente: Elaboración propia con datos de Reuters. 


\section{REFERENCIAS BIBLIOGRÁFICAS}

Addison, P. (2002), The Illustrated Wavelet Transform Handbook, Taylor and Francis, Reino Unido, $1^{a}$ edición.

Adler, M., y D. Simon (1986), "Exchange Risk Surprises in International Portfolios", Journal of Portfolio Management, Vol. 12, pp. 44-53.

Aggrawal, R., y N. A. Kyaw (2005), "Equity Market Integration in the NAFTA Region: Evidence from Unit Root and Cointegration Tests", International Review of Financial Analysts, Vol. 4, pp. 393-406.

Atkan, B., A. B. Mabrouk, M. Ozturk, y N. Rhaiem (2009), "Wavelet-Based Systemic Risk Estimation: An Application on Istanbul Stock Exchange", International Research Journal of Finance and Economics, núm. 23, pp. 33-45.

Behrad, N. (2008), "Portfolio Allocation using Wavelet Transform", disertación doctoral, The City University of New York, Facultad de Economía.

Berben, R. P., y W. J. Jansen (2005), “Comovement in International Equity Markets: A Sectoral View", North American Journal of Economics and Finance, Vol. 8, pp. 23-37.

Black, F. (1989), “Universal Hedging: Optimizing Currency Risk and Reward in International Equity Portfolios”, Financial Analysts Journal, Vol. 45, pp. 16-22.

Boroschek, R., L. Szczecinski, D. Correa, A. Morales, y R. Rivas (2002), “Detección de propiedades tiempo-frecuencia en registros sísmicos chilenos", VIII Jornadas Chilenas de Sismología e Ingeniería Antisísmica, Valparaíso, Chile.

Brooks, R., y M. del Negro (2004), "The Rise in Comovement Across National Stock Markets: Market Integration or IT Bubble”, Journal of Empirical Finance, Vol. 11, pp. 659-680.

Burrus, C. S., R. A. Gopinath, y H. Guo (1998), Introduction to Wavelets and Wavelet Transforms, A Primer, Nueva Jersey, Prentice Hall.

Ciner, C. (2006), "A Further Look at Linkages Between NAFTA Equity Markets", The Quarterly Review of Economics and Finance, Vol. 46, núm. 3, pp. 338-352.

Chukwuogor-Ndu, C., y K. Kasibhatla (2007), "Post NAFTA Integration of North American Stock Markets: Implications for Financial Decision Making", North American Journal of Finance and Banking Research, Vol. 1, núm. 1, pp. 37-53.

Darrat, A. F., y M. Zhong (2005), "Equity Market Linkage and Multinational Trade Accords: The Case of NAFTA", Journal of International Money and Finance, Vol. 24, pp. 793-817.

Daubechies, I. (1988), “Orthonormal Bases of Compactly Supported Wavelets”, Communications on Pure and Applied Mathematics, núm. 41, pp. 909-996. 
Eun, C., y B. Resnick (1984), "Estimating the Correlation Structure of International Share Prices", Journal of Finance, Vol. 39, núm. 5, pp. 1311-1324.

(1988), "Exchange Rate Uncertainty, Forward Contracts and International Portfolio Selection", Journal of Finance, Vol. 43, núm. 1, pp. 197-215.

Fadhlaoui, K., M. Bellalah, A. Dherry, y M. Zouaouii (2009), “An Empirical Examination of International Diversification Benefits in Central European Emerging Equity Markets", International Journal of Business, Vol. 14, núm. 2, pp. 163 173.

Fernández, V. (2005), "The Intertemporal CAPM and a Wavelet-Based Decomposition of Value at Risk", Studies in Nonlinear Dynamics and Econometrics, núm. 9, pp. 1-35.

Flavin, T. J. (2004), "The Effect of the Euro on Country versus Industry Portfolio Diversification", Journal of International Money and Finance, Vol. 23, pp. 11371158.

Forbes, K., y R. Rigobon (1999), "No Contagion, Only Interdependence: Measuring Stock Market Co-movements", NBER Working Paper Series, núm. 7267.

French, K., y J. Poterba (1991), "Investor Diversification and International Equity Markets", American Economic Review, Vol. 81, núm. 2, pp. 222-226.

Gencay, R., F. Selcuk, y B. Whitcher (2002), An Introduction to Wavelets and Other Filtering Methods in Finance and Economics, Academic Press, EUA.

Graps, A. (1995), “An Introduction to Wavelets", IEEE Proceedings Computational Science and Engineering, núm. 2, pp. 50-61.

Griffin, J. M., y A. G. Karolyi (1998), “Another Look at Industrial Structure of Market for International Diversification”, Journal of Financial Economics, Vol. 50, pp. 321-344.

Grinsted, A., J. C. Moore, y S. Jevrejeva (2004), “Application of the Cross Wavelet Transform and Wavelet Coherence to Geophysical Time Series", Nonlinear Processes in Geophysics, núm. 11, pp. 561-566.

Grubel, H. (1968), "Internationally Diversified Portfolios: Welfare Gains and Capital Flows", American Economic Review, Vol. 58, núm. 5, pp. 1299-1314.

Härdle, W., G. Kerkyacharian, D. Picard, y A. Tsybakov (1998), "Wavelets, Approximation and Statistical Applications", Lecture Notes in Statistics, núm. 129, primavera.

Kanas, A. (1998), "Linkages Between the US and European Equity Markets: Further Evidence from Cointegration Tests", Applied Financial Economics, Vol. 8, pp. 245-256. 
Lai, K., K. He, C. Xie, y S. Chen (2006), "Market Risk for Nonferrous Metals: a Wavelet Based VaR Approach", IEEE Proceedings of the Sixth International Conference on Intelligent Systems Design and Applications, pp. 356-362.

Lee, H. S. (2004), "International Transmission of Stock Market Movements: A Wavelet Analysis", Applied Economics Letters, Vol. 11, núm. 3, 197-201.

Levy, H., y M. Sarnat, (1970), "International Diversification of Investment Portfolios", American Economic Review, Vol. 60, núm. 4, pp. 668-675.

Mallat, S. (1989), "A Theory for Multiresolution Signal Decomposition: The Wavelet Representation”, IEEE Transactions on Pattern Analysis and Machine Intelligence, núm. 11, pp. 674-693.

Markowitz, H. (1952), “Portfolio Selection”, The Journal of Finance, Vol. 7, núm. 1, pp. 77-91.

Nelsen, Roger (2007), An Introduction to Copulas, Nueva York, Springer Series in Statistics, pp. 270.

Norsworthy, J., D. Li, y R. Gorener (2000), “Wavelet-Based Analysis of Time Series: An Export from Engineering to Finance", IEEE Proceedings Engineering Management Society, pp. 126-132.

Ramsey, J., y C. Lampart (1997), "The Decomposition of Economic Relationships by Time Scale Using Wavelets", C.V. Starr Center for Applied Economics, Working Paper, núm. 97-08, New York University. Disponible en internet: http:// ideas.repec.org/p/cvs/starer/97-08.html.

(1999), "The Decomposition of Economic Relationships by Time Scale Using Wavelets: Expenditure and Income", Studies in Nonlinear Dynamics and Econometrics, núm. 3, pp. 23-42.

Ranta, M. (2008), "Correlation Structure of Security Markets: A Wavelet Approach", Proceedings of International Conference on Applied Business \& Economics, Grecia.

(2010), "Wavelet Multiresolution Analysis of Financial Time Series", PhD dissertation, University of Vaasa, Faculty of Technology. Disponible en internet: http://www.uwasa.fi/materiaali/pdf/isbn_978-952-476-303-5.pdf.

Riley, K. F., M. P. Hobson, y S. J. Bence (1997), Mathematical Methods for Physics and Engineering, Reino Unido, Cambridge University Press.

Rua, A., y L. C. Nunes (2009), "International Comovement of Stock Market Returns: A Wavelet Analysis", Journal of Empirical Finance, Vol. 16, núm. 4, pp. 632639.

Ruppert, D. (2004), Statistics and Finance: An Introduction, Springer Texts in Statistics, Nueva York, Springer-Verlag. 
82 ECONOMÍA: TEORÍA Y PRÁCTICA • Nueva Época, número 32, enero-junio 2010

Serroukh, A., A. Walden, y D. B. Percival (2000), "Statistical Properties and Uses of the Wavelet Variance Estimator for the Scale Analysis of Time Series", Journal of the American Statistical Association, Vol. 95, núm. 449, pp. 184-196.

Sharkasi, A., H. J. Ruskin, y M. Crane, (2005), "Interrelationships among International Stock Market Indices: Europe, Asia and the Americas", International Journal of Theoretical and Applied Finance, Vol. 8, núm. 5, pp. 603-622.

Solnik, B. (1974), “Why Not Diversify Internationally?", Financial Analysts Journal, Vol. 20, pp. 48-54.

Téllez, J. C. (2009), "Estimación del valor en riesgo a través de wavelets", disertación doctoral, Escuela de Graduados en Administración y Dirección de Empresas, ITESM-campus Ciudad de México.

— , T. Vargas, y J. A. Hernández (2009), "Estimating Market Risk under a Wavelet-Based Approach: Mexican Case”, ICCSIT, 2nd IEEE International Conference on Computer Science and Information Technology, pp. 353-357.

Xiong, X., X. Zhang, W. Zhang, W., y C. Li (2005), “Wavelet-Based Beta Estimation of China Stock Market", IEEE Proceedings Fourth International Conference on Machine Learning and Cybernetics, núm. 6, pp. 3501-3505. 